\title{
Modeling a Tumor Growth with Piecewise Constant Arguments
}

\author{
F. Bozkurt \\ Department of Mathematics, Faculty of Education, Erciyes University, 38039 Kayseri, Turkey \\ Correspondence should be addressed to F. Bozkurt; fbozkurt@erciyes.edu.tr
}

Received 22 February 2013; Accepted 18 April 2013

Academic Editor: Qingdu Li

Copyright (C) 2013 F. Bozkurt. This is an open access article distributed under the Creative Commons Attribution License, which permits unrestricted use, distribution, and reproduction in any medium, provided the original work is properly cited.

\begin{abstract}
This study is based on an early brain tumor growth that is modeled as a hybrid system such as (A): $d x(t) / d t=x(t)\{r(1-\alpha x(t)-$ $\left.\left.\beta_{0} x(\llbracket t \rrbracket)-\beta_{1} x(\llbracket t-1 \rrbracket)\right)+\gamma_{1} x(\llbracket t \rrbracket)+\gamma_{2} x(\llbracket t-1 \rrbracket)\right\}$, where the parameters $\alpha, \beta_{0}, \beta_{1}$, and $r$ denote positive numbers, $\gamma_{1}$ and $\gamma_{2}$ are negative numbers and $\llbracket t \rrbracket$ is the integer part of $t \in[0, \infty)$. Equation (A) explains a brain tumor growth, where $\gamma_{1}$ is embedded to show the drug effect on the tumor and $\gamma_{2}$ is a rate that causes a negative effect by the immune system on the tumor population. Using (A), we have constructed two models of a tumor growth: one is (A) and the other one is a population model at low density by incorporating an Allee function to (A) at time $t$. To consider the global behavior of (A), we investigate the discrete solutions of (A). Examination of the characterization of the stability shows that increase of the population growth rate decreases the local stability of the positive equilibrium point of (A). The simulations give a detailed description of the behavior of solutions of (A) with and without Allee effect.
\end{abstract}

\section{Introduction}

Cancer biology has been revolutionized over the past several decades. Genetic alterations that lead to malignant phenotypes have been identified $[1,2]$, and mechanisms necessary to sustain a solid tumor [3] and that contribute to tumor-cell invasion $[4,5]$. Mathematical modeling of both tumor growth and angiogenesis has been active areas of research. Such models can be classified into one of two categories: those that analyze the remodeling of the vasculature while ignoring changes in the tumor mass and those that predict tumor expansion in the presence of a nonevolving vasculature.

The works in $[6,7]$ are very important, since they developed a two-dimensional hybrid cellular automaton model of brain tumor growth. Showing a simple model for a single species, the well-known model is constructed by May [8] and May and Oster [9] such as

$$
\frac{d x(t)}{d t}=r x(t)\left\{1-\frac{x(\llbracket t \rrbracket)}{K}\right\}
$$

who obtained that the asymptotic behavior of difference solutions can be complex and "chaotic" for certain parameter values of $r$. In recent years, several studies have been conducted to investigate the difference solutions of specific logistic differential equations with respect to the parameters [10-19]. When $\gamma_{1}=\gamma_{2}=0$ in (A), [13] considered the logistic equation

$$
\frac{d x(t)}{d t}=r x(t)\left(1-\alpha x(t)-\beta_{0} x(\llbracket t \rrbracket)-\beta_{1} x(\llbracket t-1 \rrbracket)\right),
$$

where $t \geq 0$, the parameters $\alpha, \beta_{0}, \beta_{1}$, and $r$ denote positive numbers, and $\llbracket t \rrbracket$ denotes the integer part of $t \epsilon$ $[0, \infty)$. Here, the local asymptotic stability of the positive equilibrium point of (2) was proven by using the Linearized Stability Theorem and the global asymptotic stability by using a suitable Lyapunov function.

An investigation of (A) can be shown in [14], where it was obtained that under the condition $3 \beta_{1}>\alpha+\beta_{0}>$ $2 \alpha+\beta_{1}$ and $\gamma_{2}>\gamma_{1}$ the positive equilibrium point of $(\mathrm{A})$ is locally asymptotically stable if and only if $\left(\gamma_{2}+\gamma_{1}\right) /\left(\alpha+\beta_{0}+\right.$ $\left.\beta_{1}\right)<r<\left(\gamma_{2} / \beta_{1}\right)$. Furthermore, under specific conditions the global asymptotic stability, the semicycle, and oscillation results of the solutions of (A) were also studied.

An important research for population models was conducted in 1931, where Allee [20] demonstrated that "Allee effect" occurs when population growth rate is reduced at low population size. The logistic model assumes that percapita growth rate declines monotonically when the density 
increases; it is shown, however, that for population subject to an Allee effect, per-capita growth rate gives a humped curve increasing at low density, up to a maximum intermediate density and then declines again. Many theoretical and laboratory studies have demonstrated the importance of the Allee effect in dynamics of small populations; see, for example, [21-28]. From this reasoning, biological facts lead us to assume the Allee function as follows:

(a) if $N=0$, then $a(N)=0$; that is, there is no reproduction without partners,

(b) $a^{\prime}(N)>0$ for $N \in(0, \infty)$; that is, Allee effect decreases as density increases,

(c) $\lim _{N \rightarrow \infty} a(N)=1$; that is, Allee effect vanishes at high density [29].

In this paper, a single species population (here especially about an early brain tumor growth) is modeled such as

$$
\begin{aligned}
\frac{d x(t)}{d t}=x(t)\{r & \left(1-\alpha x(t)-\beta_{0} x(\llbracket t \rrbracket)-\beta_{1} x(\llbracket t-1 \rrbracket)\right) \\
& \left.+\gamma_{1} x(\llbracket t \rrbracket)+\gamma_{2} x(\llbracket t-1 \rrbracket)\right\},
\end{aligned}
$$

where $t \geq 0$, the parameters $\alpha, \beta_{0}, \beta_{1}$, and $r$ denote positive numbers, $\gamma_{1}, \gamma_{2}$ negative numbers, and $\llbracket t \rrbracket$ denotes the integer part of $t \in[0, \infty)$. The parameter $r$ is the population growth rate of this tumor and $\alpha, \beta_{0}$, and $\beta_{1}$ are rates for the delayed tumor volume and basis of a logistic population model. $\gamma_{1}$ is embedded to show the drug effect on the tumor and $\gamma_{2}$ is a rate that causes a negative effect by the immune system on the tumor population. In Section 2 we investigate the local and global behaviors of the nonlinear difference solutions of (3) basin under specific conditions. Additionally, a characterization of the stability when the population growth rate increases was also investigated. Section 3 gives results of the local and global asymptotic behaviors of the nonlinear difference solutions of (3) with Allee effect. The discrepancy of the stability behavior with and without Allee effect of (3) will give interesting results, which is discussed in Section 4.

\section{Local and Global Asymptotic Stability Analysis}

An integration of (A) on an interval of the form $t \in[n, n+1)$ leads to

$$
x(t)=x(n) \cdot e^{\int_{n}^{t}\left(r+\left(\gamma_{1}-\beta_{0} r\right) x(n)+\left(\gamma_{2}-\beta_{1} r\right) x(n-1)-\alpha r x(s)\right) d s} .
$$

In (4) if $x(n)>0$, then $x(t)>0$. Let $t \rightarrow n+1$; it is clear that $x(n+1)>0$. This implies that we have positive solutions of (A) for positive initial conditions.

In addition, on an interval of the form $t \in[n, n+1)$ one can write $(\mathrm{A})$ as

$$
\begin{aligned}
\frac{d x(t)}{d t} & -\left\{r+\left(\gamma_{1}-\beta_{0} r\right) x(n)+\left(\gamma_{2}-\beta_{1} r\right) x(n-1)\right\} x(t) \\
& =-\alpha r x^{2}(t) .
\end{aligned}
$$

It is well known that (5) is a Bernoulli differential equation, and so for $t \rightarrow n+1$ its solutions are

$$
\begin{aligned}
& x(n+1) \\
&= x(n) \cdot e^{\left\{r+\left(\gamma_{1}-\beta_{0} r\right) x(n)+\left(\gamma_{2}-\beta_{1} r\right) x(n-1)\right\}} \\
& \times\left(1+\alpha r x(n)\left\{\frac{e^{\left\{r+\left(\gamma_{1}-\beta_{0} r\right) x(n)+\left(\gamma_{2}-\beta_{1} r\right) x(n-1)\right\}}-1}{r+\left(\gamma_{1}-\beta_{0} r\right) x(n)+\left(\gamma_{2}-\beta_{1} r\right) x(n-1)}\right\}\right)^{-1}, \\
& n=0,1,2, \ldots,
\end{aligned}
$$

where $r+\left(\gamma_{1}-\beta_{0} r\right) x(n)+\left(\gamma_{2}-\beta_{1} r\right) x(n-1) \neq 0$. The solution of (6) does not give any information about the global behavior of the differential equation. Hence, we can continue to investigate more about (6), since (6) is the difference equation of second order. Let $\gamma_{1}=-\delta_{1}$ and $\gamma_{2}=-\delta_{2}$, where $\delta_{1}$ and $\delta_{2}$ are positive numbers. It is important to take into account that the drug therapy $\gamma_{1}$ has more destroying effect on the tumor than the immune system. That is why $\delta_{1}>\delta_{2}$. Considering (6) again, we obtain, for $n=0,1,2, \ldots$,

$$
\begin{aligned}
& x(n+1) \\
& =x(n)\left(r-\left(\delta_{1}+\beta_{0} r\right) x(n)-\left(\delta_{2}+\beta_{1} r\right) x(n-1)\right) \\
& \times\left(\left(r-\left(\delta_{1}+\beta_{0} r+\alpha r\right) x(n)-\left(\delta_{2}+\beta_{1} r\right) x(n-1)\right)\right. \\
& \quad \times \exp \left(-\left\{r-\left(\delta_{1}+\beta_{0} r\right) x(n)-\left(\delta_{2}+\beta_{1} r\right) x(n-1)\right\}\right) \\
& \quad+\operatorname{\alpha rx}(n))^{-1},
\end{aligned}
$$

where hereafter

$$
r-\left(\delta_{1}+\beta_{0} r\right) x(n)-\left(\delta_{2}+\beta_{1} r\right) x(n-1) \neq 0 .
$$

Computations reveal that the equilibrium points of (7) are

$$
\bar{x}_{1}=0, \quad \bar{x}_{2}=\frac{r}{\left(\alpha+\beta_{0}+\beta_{1}\right) r+\delta_{1}+\delta_{2}} .
$$

The fundamental study contains the stability analysis of the positive equilibrium point $\bar{x}_{2}$. For this reason we show only the characteristic equation of (7) by linearizing (7) about $\bar{x}_{2}$. Computation gives a quadratic equation such as

$$
\begin{gathered}
\mu^{2}-\left\{\frac{-\left(\delta_{1}+\beta_{0} r\right)+\left(\left(\alpha+\beta_{0}\right) r+\delta_{1}\right) \cdot e^{-A}}{\alpha r}\right\} \\
\times \mu-\left\{\frac{-\left(\delta_{2}+\beta_{1} r\right)\left(1-e^{-A}\right)}{\alpha r}\right\}=0,
\end{gathered}
$$

where $A=\alpha r^{2} /\left(\left(\alpha+\beta_{0}+\beta_{1}\right) r+\delta_{1}+\delta_{2}\right)$.

Theorem 1. Let $\beta_{0}>\beta_{1}+\alpha$ and $\beta_{1}>\alpha$. The positive equilibrium point of (7) is locally asymptotically stable if and only if

$$
A<\ln \left(\frac{\left(\beta_{0}-\beta_{1}+\alpha\right) r+\delta_{1}-\delta_{2}}{\left(\beta_{0}-\beta_{1}-\alpha\right) r+\delta_{1}-\delta_{2}}\right) .
$$


Proof. By the Linearized Stability Theorem [30] we get that the positive equilibrium point of (7) is locally asymptotically stable if and only if

$$
\begin{gathered}
\left|\frac{-\left(\delta_{1}+\beta_{0} r\right)+\left(\left(\alpha+\beta_{0}\right) r+\delta_{1}\right) \cdot e^{-A}}{\alpha r}\right| \\
<1+\frac{\left(\delta_{2}+\beta_{1} r\right)\left(1-e^{-A}\right)}{\alpha r}<2
\end{gathered}
$$

holds. We can write (12) such as

(a) $\left|\left(-\left(\delta_{1}+\beta_{0} r\right)+\left(\left(\alpha+\beta_{0}\right) r+\delta_{1}\right) \cdot e^{-A}\right) / \alpha r\right|<1+\left(\delta_{2}+\right.$ $\left.\beta_{1} r\right)\left(1-e^{-A}\right) / \alpha r$

(b) $1+\left(\delta_{2}+\beta_{1} r\right)\left(1-e^{-A}\right) / \alpha r<2$.

Since $\beta_{1}>\alpha$, from (b) we have

$$
A<\ln \left(\frac{\delta_{2}+\beta_{1} r}{\delta_{2}+\left(\beta_{1}-\alpha\right) r}\right) .
$$

From (a) we get

$$
A<\ln \left(\frac{\left(\beta_{0}-\beta_{1}+\alpha\right) r+\delta_{1}-\delta_{2}}{\left(\beta_{0}-\beta_{1}-\alpha\right) r+\delta_{1}-\delta_{2}}\right),
$$

where $\beta_{0}>\beta_{1}+\alpha$. Considering both (13) and (14), we will have

$$
\begin{aligned}
A & <\ln \left(\frac{\left(\beta_{0}-\beta_{1}+\alpha\right) r+\delta_{1}-\delta_{2}}{\left(\beta_{0}-\beta_{1}-\alpha\right) r+\delta_{1}-\delta_{2}}\right) \\
& <\ln \left(\frac{\delta_{2}+\beta_{1} r}{\delta_{2}+\left(\beta_{1}-\alpha\right) r}\right),
\end{aligned}
$$

since $\left(\beta_{0}+\alpha-\beta_{1}\right) r+\delta_{1}-\delta_{2}>0$. This completes our proof.

Theorem 2. Suppose that $r-\left(\delta_{1}+\beta_{0} r+\alpha r\right) x(n)-\left(\delta_{2}+\right.$ $\left.\beta_{1} r\right) x(n-1)>0$ for $n=0,1,2, \ldots$ and assume that the conditions in Theorem 1 hold.

If

$$
\begin{aligned}
r- & \left(\delta_{1}+\beta_{0} r\right) x(n)-\left(\delta_{2}+\beta_{1} r\right) x(n-1) \\
& <\ln \left(\frac{2 \bar{x}_{2}-x(n)}{x(n)}\right) \\
x(n) & <\frac{2 r}{\left(\alpha+\beta_{0}+\beta_{1}\right) r-\gamma_{1}-\gamma_{2}},
\end{aligned}
$$

then the positive equilibrium point of (7) is globally asymptotically stable.

Proof. We consider a Lyapunov function $V(n)$ defined by

$$
V(n)=\left\{x(n)-\bar{x}_{2}\right\}^{2}, \quad n=0,1,2, \ldots
$$

The change along the solutions of (17) is

$$
\begin{aligned}
\Delta V(n) & =V(n+1)-V(n) \\
& =\{x(n+1)-x(n)\}\left\{x(n+1)+x(n)-2 \bar{x}_{2}\right\} .
\end{aligned}
$$

Considering (18), we get

$$
x(n+1)-x(n)=\frac{U_{1}}{V},
$$

where

$U_{1}$

$$
\begin{aligned}
= & \left(1-\exp \left(-\left\{r-\left(\delta_{1}+\beta_{0} r\right) x(n)-\left(\delta_{2}+\beta_{1} r\right) x(n-1)\right\}\right)\right) \\
& \times x(n) \cdot\left(r-\left(\delta_{1}+\beta_{0} r+\alpha r\right) x(n)-\left(\delta_{2}+\beta_{1} r\right) x(n-1)\right), \\
V & \\
= & \exp \left(-\left\{r-\left(\delta_{1}+\beta_{0} r\right) x(n)-\left(\delta_{2}+\beta_{1} r\right) x(n-1)\right\}\right) \\
& \cdot\left(r-\left(\delta_{1}+\beta_{0} r+\alpha r\right) x(n)-\left(\delta_{2}+\beta_{1} r\right) x(n-1)\right) \\
& +\operatorname{\alpha rx}(n) .
\end{aligned}
$$

Furthermore, from (18) we will have

$$
x(n+1)+x(n)-2 \bar{x}_{2}=\frac{U_{2}}{V},
$$

where

$$
\begin{aligned}
& U_{2} \\
& \begin{aligned}
= & \alpha r x(n)\left(x(n)-2 \bar{x}_{2}\right) \\
& \times\left(1-\exp \left(-\left\{r-\left(\delta_{1}+\beta_{0} r\right) x(n)-\left(\delta_{2}+\beta_{1} r\right) x(n-1)\right\}\right)\right) \\
& +\left(r-\left(\delta_{1}+\beta_{0} r\right) x(n)-\left(\delta_{2}+\beta_{1} r\right) x(n-1)\right) \\
& \cdot\left(\exp \left(-\left\{r-\left(\delta_{1}+\beta_{0} r\right) x(n)-\left(\delta_{2}+\beta_{1} r\right) x(n-1)\right\}\right)\right. \\
& \cdot x(n)+x(n)-2 \bar{x}_{2} \\
& \left.\cdot \exp \left(-\left\{r-\left(\delta_{1}+\beta_{0} r\right) x(n)-\left(\delta_{2}+\beta_{1} r\right) x(n-1)\right\}\right)\right) .
\end{aligned}
\end{aligned}
$$

Since $0<r-\left(\delta_{1}+\beta_{0} r-\alpha r\right) x(n)-\left(\delta_{2}+\beta_{1} r\right) x(n-1)$, if

$$
\begin{gathered}
x(n)<\bar{x}_{2}, \\
r-\left(\delta_{1}+\beta_{0} r\right) x(n)-\left(\delta_{2}+\beta_{1} r\right) x(n-1) \\
<\ln \left(\frac{2 \bar{x}_{2}-x(n)}{x(n)}\right),
\end{gathered}
$$

then

$$
x(n+1)-x(n)>0, \quad x(n+1)+x(n)-2 \bar{x}_{2}<0 .
$$

This implies that $\Delta V(n)<0$, which gives the condition for the global asymptotic stability of the positive equilibrium point of (7).

Theorem 3. Let $r_{1}$ and $r_{2}$ be population growth rates of (7) such that $r_{1}<r_{2}$ and suppose that $\bar{x}^{*}$ and $\bar{x}^{* *}$ are positive equilibrium points of (7) with respect to $r_{1}$ and $r_{2}$ that hold 
the conditions in Theorem 1, respectively. Furthermore, assume that

$$
\ln \left(\frac{r_{2}}{r_{1}}\right)<A_{2}-A_{1},
$$

where $A_{1}=\alpha r_{1}^{2} /\left(\left(\alpha+\beta_{0}+\beta_{1}\right) r_{1}+\delta_{1}+\delta_{2}\right)$ and $A_{2}=\alpha r_{2}^{2} /((\alpha+$ $\left.\left.\beta_{0}+\beta_{1}\right) r_{2}+\delta_{1}+\delta_{2}\right)$. If

$$
\begin{aligned}
& A_{1}>\ln \left(\frac{\left(\alpha+\beta_{0}+\beta_{1}\right) r_{1}+\delta_{1}+\delta_{2}}{\left(\beta_{0}+\beta_{1}\right) r_{1}+\delta_{2}+\delta_{1}}\right), \\
& A_{2}<\ln \left(\frac{\left(\alpha+\beta_{0}+\beta_{1}\right) r_{2}+\delta_{1}+\delta_{2}}{\left(\beta_{0}+\beta_{1}\right) r_{2}+\delta_{2}+\delta_{1}}\right),
\end{aligned}
$$

then the local stability of $\bar{x}^{* *}$ is weaker than $\bar{x}^{*}$. That is, increase of the population growth rate decreases the local stability of the positive equilibrium point in (7).

Proof. Let us write from (7)

$$
\begin{aligned}
& x(n+1) \\
& =x(n)\left(r_{1}-\left(\delta_{1}+\beta_{0} r_{1}\right) x(n)-\left(\delta_{2}+\beta_{1} r_{1}\right) x(n-1)\right) \\
& \quad \times\left(\left(r_{1}-\left(\delta_{1}+\beta_{0} r_{1}+\alpha r_{1}\right) x(n)-\left(\delta_{2}+\beta_{1} r_{1}\right) x(n-1)\right)\right. \\
& \quad \times \exp \left(-\left\{r_{1}-\left(\delta_{1}+\beta_{0} r_{1}\right) x(n)-\left(\delta_{2}+\beta_{1} r_{1}\right) x(n-1)\right\}\right) \\
& \left.\quad+\alpha r_{1} x(n)\right)^{-1}, \\
& x(n+1) \\
& =x(n)\left(r_{2}-\left(\delta_{1}+\beta_{0} r_{2}\right) x(n)-\left(\delta_{2}+\beta_{1} r_{2}\right) x(n-1)\right) \\
& \quad \times\left(\left(r_{2}-\left(\delta_{1}+\beta_{0} r_{2}+\alpha r_{2}\right) x(n)-\left(\delta_{2}+\beta_{1} r_{2}\right) x(n-1)\right)\right. \\
& \quad \times \exp \left(-\left\{r_{2}-\left(\delta_{1}+\beta_{0} r_{2}\right) x(n)-\left(\delta_{2}+\beta_{1} r_{2}\right) x(n-1)\right\}\right) \\
& \left.\quad+\alpha r_{2} x(n)\right)^{-1},
\end{aligned}
$$

where $r_{1}<r_{2}$. In this case, the positive equilibrium points of (27) are

$$
\begin{aligned}
& \bar{x}^{*}=\frac{r_{1}}{\left(\alpha+\beta_{0}+\beta_{1}\right) r_{1}+\delta_{1}+\delta_{2}}, \\
& \bar{x}^{* *}=\frac{r_{2}}{\left(\alpha+\beta_{0}+\beta_{1}\right) r_{2}+\delta_{1}+\delta_{2}},
\end{aligned}
$$

respectively. The characteristic equations of (27) are

$$
\begin{gathered}
\mu^{2}-\left\{\frac{-\left(\delta_{1}+\beta_{0} r_{1}\right)+\left(\left(\alpha+\beta_{0}\right) r_{1}+\delta_{1}\right) \cdot e^{-A_{1}}}{\alpha r_{1}}\right\} \\
\times \mu-\left\{\frac{-\left(\delta_{2}+\beta_{1} r_{1}\right)\left(1-e^{-A_{1}}\right)}{\alpha r_{1}}\right\}=0,
\end{gathered}
$$

where $A_{1}=\alpha r_{1}^{2} /\left(\left(\alpha+\beta_{0}+\beta_{1}\right) r_{1}+\delta_{1}+\delta_{2}\right)$ and

$$
\begin{gathered}
\mu^{2}-\left\{\frac{-\left(\delta_{1}+\beta_{0} r_{2}\right)+\left(\left(\alpha+\beta_{0}\right) r_{2}+\delta_{1}\right) \cdot e^{-A_{2}}}{\alpha r_{2}}\right\} \\
\times \mu-\left\{\frac{-\left(\delta_{2}+\beta_{1} r_{2}\right)\left(1-e^{-A_{2}}\right)}{\alpha r_{2}}\right\}=0,
\end{gathered}
$$

where $A_{2}=\alpha r_{2}^{2} /\left(\left(\alpha+\beta_{0}+\beta_{1}\right) r_{2}+\delta_{1}+\delta_{2}\right)$, respectively. Since $r_{1}<r_{2}$, we can write

$$
\begin{aligned}
\frac{1}{\alpha r_{2}}<\frac{1}{\alpha r_{1}}, \\
\delta_{2}+\beta_{1} r_{1}<\delta_{2}+\beta_{1} r_{2} .
\end{aligned}
$$

The inequality (32) can be also written as

$$
-\left(\delta_{2}+\beta_{1} r_{2}\right)<-\left(\delta_{2}+\beta_{1} r_{1}\right) .
$$

Since the inequality

$$
\left(\alpha+\beta_{0}+\beta_{1}\right) r_{1} r_{2}\left(r_{2}-r_{1}\right)+\left(\delta_{1}+\delta_{2}\right)\left(r_{2}-r_{1}\right)\left(r_{2}+r_{1}\right)>0
$$

always holds, we get

$$
\frac{\alpha r_{2}^{2}}{\left(\alpha+\beta_{0}+\beta_{1}\right) r_{2}+\delta_{1}+\delta_{2}}>\frac{\alpha r_{1}^{2}}{\left(\alpha+\beta_{0}+\beta_{1}\right) r_{1}+\delta_{1}+\delta_{2}} .
$$

The inequality (35) can be also written as

$$
\frac{-\alpha r_{2}^{2}}{\left(\alpha+\beta_{0}+\beta_{1}\right) r_{2}+\delta_{1}+\delta_{2}}<\frac{-\alpha r_{1}^{2}}{\left(\alpha+\beta_{0}+\beta_{1}\right) r_{1}+\delta_{1}+\delta_{2}}
$$

or

$$
-A_{2}<-A_{1} .
$$

It is obvious that from (37), we get

$$
\delta_{2} e^{-A_{2}}<\delta_{2} e^{-A_{1}} .
$$

Furthermore, if

$$
\ln \left(\frac{r_{2}}{r_{1}}\right)<A_{2}-A_{1},
$$

then the inequality

$$
\beta_{1} r_{2} e^{-A_{2}}<\beta_{1} r_{1} e^{-A_{1}}
$$

holds. In view of (39), considering (31), (33), (38), and (40) together, we obtain

$$
-1<\frac{-\left(\delta_{2}+\beta_{1} r_{2}\right)\left(1-e^{-A_{2}}\right)}{\alpha r_{2}}<\frac{-\left(\delta_{2}+\beta_{1} r_{1}\right)\left(1-e^{-A_{1}}\right)}{\alpha r_{1}} .
$$

From the Linearized Stability Theorem we want to obtain the conditions that satisfy the inequality

$$
\begin{aligned}
-1< & \frac{-\left(\delta_{1}+\beta_{0} r_{2}\right)+\left(\left(\alpha+\beta_{0}\right) r_{2}+\delta_{1}\right) \cdot e^{-A_{2}}}{\alpha r_{2}} \\
& -\frac{-\left(\delta_{2}+\beta_{1} r_{2}\right)\left(1-e^{-A_{2}}\right)}{\alpha r_{2}} \\
< & \frac{-\left(\delta_{1}+\beta_{0} r_{1}\right)+\left(\left(\alpha+\beta_{0}\right) r_{1}+\delta_{1}\right) \cdot e^{-A_{1}}}{\alpha r_{1}} \\
& -\frac{-\left(\delta_{2}+\beta_{1} r_{1}\right)\left(1-e^{-A_{1}}\right)}{\alpha r_{1}} .
\end{aligned}
$$


Simplifications of (42) give us the inequality

$$
\begin{aligned}
& \frac{\left(\left(\beta_{1}-\beta_{0}\right) r_{2}+\delta_{2}-\delta_{1}\right)+\left(\left(\alpha+\beta_{0}-\beta_{1}\right) r_{2}+\delta_{1}-\delta_{2}\right) \cdot e^{-A_{2}}}{\alpha r_{2}} \\
& <\frac{\left(\left(\beta_{1}-\beta_{0}\right) r_{1}+\delta_{2}-\delta_{1}\right)+\left(\left(\alpha+\beta_{0}-\beta_{1}\right) r_{1}+\delta_{1}-\delta_{2}\right) \cdot e^{-A_{1}}}{\alpha r_{1}} .
\end{aligned}
$$

Since $\beta_{0}>\alpha+\beta_{1}$ and $\delta_{1}>\delta_{2}$, we get

$$
\begin{gathered}
\left(\left(\alpha+\beta_{0}-\beta_{1}\right) r_{2}+\delta_{1}-\delta_{2}\right) \cdot e^{-A_{2}} \\
<\left(\left(\alpha+\beta_{0}-\beta_{1}\right) r_{1}+\delta_{1}-\delta_{2}\right) \cdot e^{-A_{1}}, \\
\left(\beta_{1}-\beta_{0}\right) r_{2}+\delta_{2}-\delta_{1}<\left(\beta_{1}-\beta_{0}\right) r_{1}+\delta_{2}-\delta_{1} .
\end{gathered}
$$

Considering both (44) and (45), we get (42).

Furthermore, from the Linearized Stability Theorem we must also show that the inequality

$$
\begin{gathered}
\frac{-\left(\delta_{1}+\beta_{0} r_{1}\right)+\left(\left(\alpha+\beta_{0}\right) r_{1}+\delta_{1}\right) \cdot e^{-A_{1}}}{\alpha r_{1}} \\
-\frac{\left(\delta_{2}+\beta_{1} r_{1}\right)\left(1-e^{-A_{1}}\right)}{\alpha r_{1}} \\
<\frac{-\left(\delta_{1}+\beta_{0} r_{2}\right)+\left(\left(\alpha+\beta_{0}\right) r_{2}+\delta_{1}\right) \cdot e^{-A_{2}}}{\alpha r_{2}} \\
-\frac{\left(\delta_{2}+\beta_{1} r_{2}\right)\left(1-e^{-A_{2}}\right)}{\alpha r_{2}}<1
\end{gathered}
$$

holds. Simplifying (46), we can write

$$
\begin{aligned}
& -\left(\left(\beta_{0}+\beta_{1}\right) r_{1}+\delta_{2}+\delta_{1}\right) \\
& +\left(\left(\alpha+\beta_{0}+\beta_{1}\right) r_{1}+\delta_{1}+\delta_{2}\right) \cdot e^{-A_{1}}\left(\alpha r_{1}\right)^{-1} \\
& \quad<-\left(\left(\beta_{0}+\beta_{1}\right) r_{2}+\delta_{2}+\delta_{1}\right) \\
& \quad+\left(\left(\alpha+\beta_{0}+\beta_{1}\right) r_{2}+\delta_{1}+\delta_{2}\right) \cdot e^{-A_{2}}\left(\alpha r_{2}\right)^{-1} .
\end{aligned}
$$

If

$$
\begin{aligned}
& A_{1}>\ln \left(\frac{\left(\alpha+\beta_{0}+\beta_{1}\right) r_{1}+\delta_{1}+\delta_{2}}{\left(\beta_{0}+\beta_{1}\right) r_{1}+\delta_{2}+\delta_{1}}\right), \\
& A_{2}<\ln \left(\frac{\left(\alpha+\beta_{0}+\beta_{1}\right) r_{2}+\delta_{1}+\delta_{2}}{\left(\beta_{0}+\beta_{1}\right) r_{2}+\delta_{2}+\delta_{1}}\right),
\end{aligned}
$$

then

$$
\begin{aligned}
& -\left(\left(\beta_{0}+\beta_{1}\right) r_{1}+\delta_{2}+\delta_{1}\right) \\
& \quad+\left(\left(\alpha+\beta_{0}+\beta_{1}\right) r_{1}+\delta_{1}+\delta_{2}\right) \cdot e^{-A_{1}}<0, \\
& -\left(\left(\beta_{0}+\beta_{1}\right) r_{2}+\delta_{2}+\delta_{1}\right) \\
& \quad+\left(\left(\alpha+\beta_{0}+\beta_{1}\right) r_{2}+\delta_{1}+\delta_{2}\right) \cdot e^{-A_{2}}>0 .
\end{aligned}
$$

The inequalities (49) imply that (46) holds. This result explains that increase of the population growth rate decreases the local stability of the positive equilibrium point in (7), which completes our proof.

Theorem 4. Let $\{x(n)\}_{n=0}^{\infty}$ be a positive solution of (7). Assume that for $n=0,1, \ldots$ the condition

$$
0<r-\left(\delta_{1}+\beta_{0} r+\alpha r x(n)\right) x(n)-\left(\delta_{2}+\beta_{1} r\right) x(n-1)
$$

holds. Then all positive solutions of (7) are in the interval

$$
x(n) \in\left(0, \frac{1}{\alpha}\right) .
$$

Proof. Let (50) hold. Then we can write

$$
r-\left(\delta_{1}+\beta_{0} r\right) x(n)-\left(\delta_{2}+\beta_{1} r\right) x(n-1)<r .
$$

From (52), we will have

$$
e^{-\left(r-\left(\delta_{1}+\beta_{0} r\right) x(n)-\left(\delta_{2}+\beta_{1} r\right) x(n-1)\right)}>e^{-r} .
$$

Considering (52) and (53) together, we get

$$
\begin{aligned}
& x(n+1) \\
& =x(n)\left(r-\left(\delta_{1}+\beta_{0} r\right) x(n)-\left(\delta_{2}+\beta_{1} r\right) x(n-1)\right) \\
& \quad \times\left(\left(r-\left(\delta_{1}+\beta_{0} r+\alpha r\right) x(n)-\left(\delta_{2}+\beta_{1} r\right) x(n-1)\right)\right. \\
& \quad \times \exp \left(-\left\{r-\left(\delta_{1}+\beta_{0} r\right) x(n)-\left(\delta_{2}+\beta_{1} r\right) x(n-1)\right\}\right) \\
& \quad+\alpha r x(n))^{-1} \\
& <x(n) r \\
& \quad \times\left(\left(r-\left(\delta_{1}+\beta_{0} r+\alpha r\right) x(n)-\left(\delta_{2}+\beta_{1} r\right) x(n-1)\right)\right. \\
& \quad \times \exp (-r)+\alpha r x(n))^{-1} .
\end{aligned}
$$

Furthermore, since we have

$$
0<\alpha r x(n)<r-\left(\delta_{1}+\beta_{0} r\right) x(n)-\left(\delta_{2}+\beta_{1} r\right) x(n-1)<r,
$$

we obtain

$$
\begin{aligned}
& x(n+1) \\
& <\frac{x(n) r}{-\alpha r x(n) \exp (-r)+\alpha r x(n) \exp (-r)+\alpha r x(n)}=\frac{1}{\alpha} .
\end{aligned}
$$

This completes the proof.

\section{Local and Global Asymptotic Stability Analysis with Allee Effect}

In this section we use an Allee function of time $t$. Let (3) be written as

$$
\frac{1}{x} \frac{d x}{d t}=r-\left(\delta_{1}+\delta_{2}+\left(\alpha+\beta_{0}+\beta_{1}\right) r\right) x,
$$


where $\gamma_{1}=-\delta_{1}$ and $\gamma_{2}=-\delta_{2}$. Applying to (57) an Allee function

$$
a(x)=\frac{x}{E+x},
$$

where $E$ is an Allee constant, we get

$$
\frac{1}{x} \frac{d x}{d t}=a(x)\left\{r-\left(\delta_{1}+\delta_{2}+\left(\alpha+\beta_{0}+\beta_{1}\right) r\right) x\right\} .
$$

By defining

$$
g(x)=a(x)\left\{r-\left(\delta_{1}+\delta_{2}+\left(\alpha+\beta_{0}+\beta_{1}\right) r\right) x\right\}
$$

and taking the derivative of $\mathrm{g}$ with respect to $x$, we obtain

$$
\begin{aligned}
g^{\prime}(x)= & -\left(\delta_{1}+\delta_{2}+\left(\alpha+\beta_{0}+\beta_{1}\right) r\right) x^{2} \\
& -2 E\left(\delta_{1}+\delta_{2}+\left(\alpha+\beta_{0}+\beta_{1}\right) r\right) x \\
& +E r(E+x)^{-2} .
\end{aligned}
$$

By showing the sign of (61), we get that $g$ is an increasing function for

$$
\begin{aligned}
x \in(0, & \left(E^{2}\left(\delta_{1}+\delta_{2}+\left(\alpha+\beta_{0}+\beta_{1}\right) r\right)^{2}\right. \\
& \left.+\operatorname{Er}\left(\left(\alpha+\beta_{0}+\beta_{1}\right) r+\delta_{1}+\delta_{2}\right)\right)^{-1 / 2} \\
- & E\left(\left(\alpha+\beta_{0}+\beta_{1}\right) r+\delta_{1}+\delta_{2}\right) \\
\times & \left.\left(\left(\alpha+\beta_{0}+\beta_{1}\right) r+\delta_{1}+\delta_{2}\right)^{-1}\right)
\end{aligned}
$$

and $g$ is a decreasing function for

$$
\begin{aligned}
x \in(( & E^{2}\left(\delta_{1}+\delta_{2}+\left(\alpha+\beta_{0}+\beta_{1}\right) r\right)^{2} \\
& \left.+E r\left(\left(\alpha+\beta_{0}+\beta_{1}\right) r+\delta_{1}+\delta_{2}\right)\right)^{-1 / 2} \\
& -E\left(\left(\alpha+\beta_{0}+\beta_{1}\right) r+\delta_{1}+\delta_{2}\right) \\
& \left.\times\left(\left(\alpha+\beta_{0}+\beta_{1}\right) r+\delta_{1}+\delta_{2}\right)^{-1}, \infty\right) .
\end{aligned}
$$

This also means that if the density is

$$
\begin{aligned}
x<( & E^{2}\left(\delta_{1}+\delta_{2}+\left(\alpha+\beta_{0}+\beta_{1}\right) r\right)^{2} \\
& \left.+E r\left(\left(\alpha+\beta_{0}+\beta_{1}\right) r+\delta_{1}+\delta_{2}\right)\right)^{-1 / 2} \\
& -E\left(\left(\alpha+\beta_{0}+\beta_{1}\right) r+\delta_{1}+\delta_{2}\right) \\
& \times\left(\left(\alpha+\beta_{0}+\beta_{1}\right) r+\delta_{1}+\delta_{2}\right)^{-1},
\end{aligned}
$$

then a population model without an Allee function will not give realistic results. But if

$$
\begin{aligned}
x> & \left(E^{2}\left(\delta_{1}+\delta_{2}+\left(\alpha+\beta_{0}+\beta_{1}\right) r\right)^{2}\right. \\
& \left.+E r\left(\left(\alpha+\beta_{0}+\beta_{1}\right) r+\delta_{1}+\delta_{2}\right)\right)^{-1 / 2} \\
& -E\left(\left(\alpha+\beta_{0}+\beta_{1}\right) r+\delta_{1}+\delta_{2}\right) \\
& \times\left(\left(\alpha+\beta_{0}+\beta_{1}\right) r+\delta_{1}+\delta_{2}\right)^{-1},
\end{aligned}
$$

then it is not important to use a model with an Allee function as it is also explained in the introduction. Applying to (3) an Allee function such as

$$
a(x(\llbracket t \rrbracket))=\frac{x(\llbracket t \rrbracket)}{E+x(\llbracket t \rrbracket)},
$$

where $E>0$, we obtain

$$
\begin{aligned}
& \frac{d x(t)}{d t} \\
& =x(t)\left\{r\left(1-\alpha x(t)-\beta_{0} x(\llbracket t \rrbracket)-\beta_{1} x(\llbracket t-1 \rrbracket)\right)\right. \\
& \left.\quad-\delta_{1} x(\llbracket t \rrbracket)-\delta_{2} x(\llbracket t-1 \rrbracket)\right\} \frac{x(\llbracket t \rrbracket)}{E+x(\llbracket t \rrbracket)} .
\end{aligned}
$$

It is clear that (67) is a Bernoulli differential equation on the interval $t \in[n, n+1)$. Solving (67) for $t \in[n, n+1)$ and $t \rightarrow n+1$, we get for $n=0,1,2, \ldots$

$$
\begin{aligned}
& x(n+1) \\
& =x(n)\left(r-\left(\delta_{1}+\beta_{0} r\right) x(n)-\left(\delta_{2}+\beta_{1} r\right) x(n-1)\right) \\
& \times\left(\left(r-\left(\delta_{1}+\beta_{0} r+\alpha r\right) x(n)-\left(\delta_{2}+\beta_{1} r\right) x(n-1)\right)\right. \\
& \quad \times \exp (-a(x(n))) \\
& \quad \times\left\{r-\left(\delta_{1}+\beta_{0} r\right) x(n)-\left(\delta_{2}+\beta_{1} r\right) x(n-1)\right\} \\
& \quad+\alpha r x(n))^{-1} .
\end{aligned}
$$

It can be shown that the equilibrium points of (68) are also the equilibrium points of (7).

Linearizing (68) about the positive equilibrium point, we obtain the characteristic equation as follows:

$$
\begin{gathered}
\mu^{2}-\left\{\frac{-\left(\delta_{1}+\beta_{0} r\right)+\left(\left(\alpha+\beta_{0}\right) r+\delta_{1}\right) \cdot e^{-a(\bar{x}) A}}{\alpha r}\right\} \\
\times \mu-\left\{\frac{-\left(\delta_{2}+\beta_{1} r\right)\left(1-e^{-a(\bar{x}) A}\right)}{\alpha r}\right\}=0,
\end{gathered}
$$

where $A=\alpha r^{2} /\left(\left(\alpha+\beta_{0}+\beta_{1}\right) r+\delta_{1}+\delta_{2}\right)$.

Theorem 5. Let $\beta_{0}>\beta_{1}+\alpha$ and $\beta_{1}>\alpha$. The positive equilibrium point of (68) is locally asymptotically stable if

$$
A<\frac{1}{a(\bar{x})} \ln \left(\frac{\left(\beta_{0}-\beta_{1}+\alpha\right) r+\delta_{1}-\delta_{2}}{\left(\beta_{0}-\beta_{1}-\alpha\right) r+\delta_{1}-\delta_{2}}\right) .
$$

Proof. The proof is similar to that in Theorem 1 and will be omitted.

Theorem 6. Suppose that $r-\left(\delta_{1}+\beta_{0} r+\alpha r\right) x(n)-\left(\delta_{2}+\right.$ $\left.\beta_{1} r\right) x(n-1)>0$ for $n=0,1,2, \ldots$ and assume that the conditions in Theorem 5 hold. 
If

$$
\begin{aligned}
r- & \left(\delta_{1}+\beta_{0} r\right) x(n)-\left(\delta_{2}+\beta_{1} r\right) x(n-1) \\
& <\ln \left(\frac{2 \bar{x}_{2}-x(n)}{x(n)}\right) \\
x(n) & <\frac{2 r}{\left(\alpha+\beta_{0}+\beta_{1}\right) r-\gamma_{1}-\gamma_{2}},
\end{aligned}
$$

then the positive equilibrium point of (68) is globally asymptotically stable.

Proof. The proof is similar to that in Theorem 2 and will be omitted.

Example 7. The goal of this investigation is to examine the development of monoclonal tumors under the effects of treatment. In view of [6], the carrying capacity of a monoclonal tumor is ca. $38 \mathrm{~mm}$. We select $\alpha^{\prime}=0.00744, \beta_{1}^{\prime}=0.007448$, and $\beta_{0}^{\prime}=0.014896$. By dividing these values by the carrying capacity, we obtain $\alpha=(0.00744 / 38)=0.000195789$, $\beta_{1}=(0.007448 / 38)=0.000196$ and $\beta_{0}=(0.014896 / 38)=$ 0.000392 . These values are also suitable for the hypotheses in Theorems 1 and 5. To have a compatible result, a relation between the model and the data is constructed by multiplying these values with 10. Thus, the parameters for (7) are in this case $\alpha=0.00195789, \beta_{1}=0.00196$, and $\beta_{0}=0.00392$. For a therapy of ca. $75 \mathrm{mg}$ drug and under the assumption that the effect on the tumor is $1.6 \%$ we obtain $\delta_{1}=0.0125$. The effect on the immune is ca. $10 \%$ compared with the effect of the drug treatment, so we have $\delta_{2}=0.00125$. Figure 1 shows us the behavior of the solution of (7). Differently from this, we can see Figure 2, where we have used the Allee function for $E=0.4$. Studies demonstrated that Allee effects play an important role in the stability analysis of equilibrium points of a population dynamics model. Generally, an Allee effect has a stabilizing effect on population dynamics. So, in (68) our expectation is that the chaos begins later as it can be also shown in Figure 2.

\section{Discussion}

Section 2 was constructed to obtain specific conditions for local and global asymptotic stability of the positive equilibrium point of (7) without Allee effect by applying the Linearized Stability Theorem and the theory of the use of a Lyapunov function, respectively. Furthermore, we showed that increase of the population growth rate decreases the stability of the positive equilibrium point of (7), which is given in Theorem 3. Finally, in Section 2 we proved Theorem 4, which has given information about the bound of the solutions of (7). By using the data of [6] about the radius of tumor at 111 days, we take the radius as $4.96 \mathrm{~mm}$ for a population growth rate $r=0.31$. By multiplying it with 10, we use the growth rate $r=3.1$. The volume of such a tumor will be then $(n)=$ $510.87 \mathrm{~mm}^{3}$. Considering Theorem 4, we can see that the above mentioned value for $\alpha$ is suitable. For $\alpha=0.00195789$,

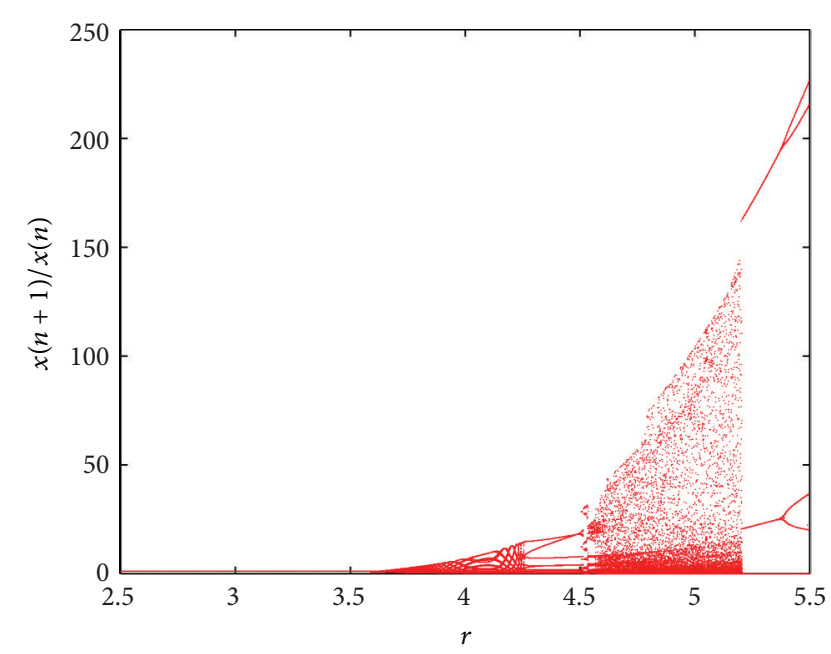

FIGURE 1: Behavior of the solutions of (7), where $\alpha=0.00195789$, $\beta_{1}=0.00196, \beta_{0}=0.00392, \delta_{1}=0.0125$, and $\delta_{2}=0.00125$.

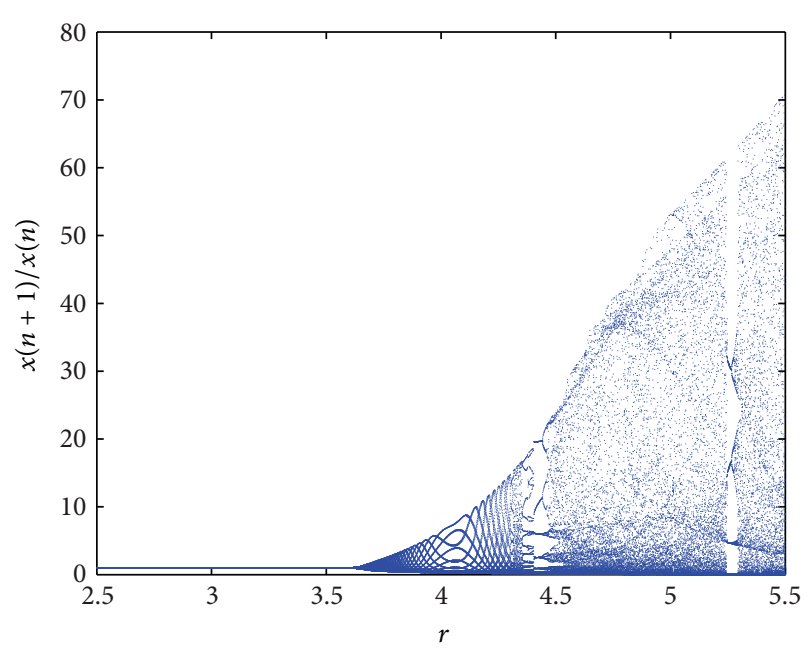

FIGURE 2: Behavior of the solutions of (68), where $\alpha=0.00195789$, $\beta_{1}=0.00196, \beta_{0}=0.00392, \delta_{1}=0.0125, \delta_{2}=0.00125$, and $E=0.4$.

$\beta_{1}=0.00196, \beta_{0}=0.00392, \delta_{1}=0.0125$, and $\delta_{2}=0.00125$ we consider Section 3 in view of $E=0.4$,

$$
\begin{aligned}
x \in(0, & \left(E^{2}\left(\delta_{1}+\delta_{2}+\left(\alpha+\beta_{0}+\beta_{1}\right) r\right)^{2}\right. \\
& \left.+\operatorname{Er}\left(\left(\alpha+\beta_{0}+\beta_{1}\right) r+\delta_{1}+\delta_{2}\right)\right)^{-1 / 2} \\
- & E\left(\left(\alpha+\beta_{0}+\beta_{1}\right) r+\delta_{1}+\delta_{2}\right) \\
\times & \left.\left(\left(\alpha+\beta_{0}+\beta_{1}\right) r+\delta_{1}+\delta_{2}\right)^{-1}\right)=(0,5.322) .
\end{aligned}
$$

In this case, for the volume $x(n)=5 \mathrm{~mm}^{3}$ the radius of the tumor must be around $1.0609 \mathrm{~mm}$, which the temporal development of a cross-central section of a tumor growing without angiogenesis show a tumor more than at day 80 and the temporal development of a cross-central section of a tumor growing with angiogenesis around 40 days of the tumor (see [6]). This means that during the 90 days of 
the tumor we shall use the model given in (68). For days more than 90, both models are suitable ((7) and (68)). Using the above mentioned values, it can be shown that the local stability of both theorems (Theorems 1 and 5) hold. However, Theorem 5 give us that the stable interval for the growth rate is wide as assumed in Theorem 1, which is important for the drug therapy.

\section{References}

[1] E. Hulleman and K. Helin, "Molecular mechanisms in gliomagenesis," Advances in Cancer Research, vol. 94, no. 1, pp. 1-27, 2005.

[2] E. A. Maher, F. B. Furnari, R. M. Bachoo et al., "Malignant glioma: genetics and biology of a grave matter," Genes and Development, vol. 15, no. 11, pp. 1311-1333, 2001.

[3] D. J. Brat, B. Kaur, and E. G. Van Meir, "Genetic modulation of hypoxia induced gene expression and angiogenesis: relevance to brain tumors," Frontiers in Bioscience, vol. 8, pp. d100-d116, 2003.

[4] A. Giese and M. Westphal, "Glioma invasion in the central nervous system," Neurosurgery, vol. 39, no. 2, pp. 235-252, 1996.

[5] T. Visted, P. O. Enger, M. Lund-Johansen, and R. Bjerkvig, "Mechanisms of tumor cell invasion and angiogenesis in the central nervous system," Frontiers in Bioscience, vol. 8, pp. e289e304, 2003.

[6] J. L. Gevertz and S. Torquato, "Modeling the effects of vasculature evolution on early brain tumor growth," Journal of Theoretical Biology, vol. 243, no. 4, pp. 517-531, 2006.

[7] J. E. Schmitz, A. R. Kansal, and S. Torquato, "A cellular automaton model of brain tumor treatment and resistance," Journal of Theoretical Medicine, vol. 4, no. 4, pp. 223-239, 2002.

[8] R. M. May, "Biological populations obeying difference equations: stable points, stable cycles, and chaos," Journal of Theoretical Biology, vol. 51, no. 2, pp. 511-524, 1975.

[9] R. M. May and G. F. Oster, "Bifurcations and dynamics complexity in simple ecological models," The American Naturalist, vol. 110, pp. 573-599, 1976.

[10] K. Gopalsamy and P. Liu, "Persistence and global stability in a population model," Journal of Mathematical Analysis and Applications, vol. 224, no. 1, pp. 59-80, 1998.

[11] P. Liu and K. Gopalsamy, "Global stability and chaos in a population model with piecewise constant arguments," Applied Mathematics and Computation, vol. 101, no. 1, pp. 63-88, 1999.

[12] K. L. Cooke and W. Huang, "A theorem of george seifert and an equation with State-Dependent Delay," in Delay and Differential Equations, A. M. Fink, R. K. Miller, and W. Kliemann, Eds., World Scientific, Ames, Iowa, USA, 1991.

[13] F. Gurcan and F. Bozkurt, "Global stability in a population model with piecewise constant arguments," Journal of Mathematical Analysis and Applications, vol. 360, no. 1, pp. 334-342, 2009.

[14] I. Ozturk and F. Bozkurt, "Stability analysis of a population model with piecewise constant arguments," Nonlinear Analysis: Real World Applications, vol. 12, no. 3, pp. 1532-1545, 2011.

[15] K. Gobalsamy, Stability and Oscillation in Delay Differential Equations of Population Dynamics, Kluwer Academic Publishers, Dodrecht, The Netherlands, 1992.

[16] J. W.-H. So and J. S. Yu, "Global stability in a logistic equation with piecewise constant arguments," Hokkaido Mathematical Journal, vol. 24, no. 2, pp. 269-286, 1995.
[17] P. Cull, “Global stability of population models," Bulletin of Mathematical Biology, vol. 43, no. 1, pp. 47-58, 1981.

[18] K. Uesugi, Y. Muroya, and E. Ishiwata, "On the global attractivity for a logistic equation with piecewise constant arguments," Journal of Mathematical Analysis and Applications, vol. 294, no. 2, pp. 560-580, 2004.

[19] Y. Muroya, "Persistence, contractivity and global stability in logistic equations with piecewise constant delays," Journal of Mathematical Analysis and Applications, vol. 270, no. 2, pp. 602$635,2002$.

[20] W. C. Allee, Animal Aggregations: A Study in General Sociology, University of Chicago Press, Chicago, Ill, USA, 1931.

[21] G. Wang, X. G. Liang, and F. Z. Wang, "The competitive dynamics of populations subject to an Allee effect," Ecological Modelling, vol. 124, no. 2-3, pp. 183-192, 1999.

[22] M. A. Asmussen, "Density-dependent selection II. The Allee effect," The American Naturalist, vol. 114, pp. 796-809, 1979.

[23] R. Lande, "Extinction thresholds in demographic models of territorial populations," American Naturalist, vol. 130, no. 4, pp. 624-635, 1987.

[24] B. Dennis, "Allee effects: population growth, critical density, and the chance of extinction," Natural Resource Modeling, vol. 3, no. 4, pp. 481-538, 1989.

[25] T. Stephan and C. Wissel, "Stochastic extinction models discrete in time," Ecological Modelling, vol. 75-76, pp. 183-192, 1994.

[26] I. Hanski, "Single-species metapopulation dynamics: concepts, models and observations," Biological Journal of the Linnean Society, vol. 42, no. 1-2, pp. 17-38, 1991.

[27] P. Amarasekare, "Allee effects in metapopulation dynamics," American Naturalist, vol. 152, no. 2, pp. 298-302, 1998.

[28] A. P. Dobson and A. M. Lyles, "The population dynamics and conservation of primate populations," Conservation Biology, vol. 3, no. 4, pp. 362-380, 1989.

[29] C. Çelik, H. Merdan, O. Duman, and O. Akın, "Allee effects on population dynamics with delay," Chaos, Solitons \& Fractals, vol. 37, no. 1, pp. 65-74, 2008.

[30] C. H. Gibbons, M. R. S. Kulenović, G. Ladas, and H. D. Voulov, "On the trichotomy character of $x_{n+1}=\left(\alpha+\beta x_{n}+\gamma x_{n-1}\right) /(A+$ $\left.x_{n}\right)$," Journal of Difference Equations and Applications, vol. 8, no. 1, pp. 75-92, 2002. 


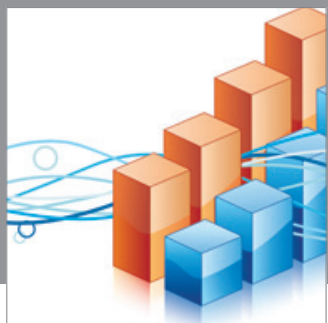

Advances in

Operations Research

mansans

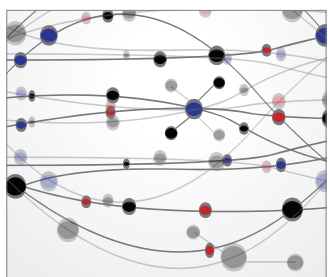

The Scientific World Journal
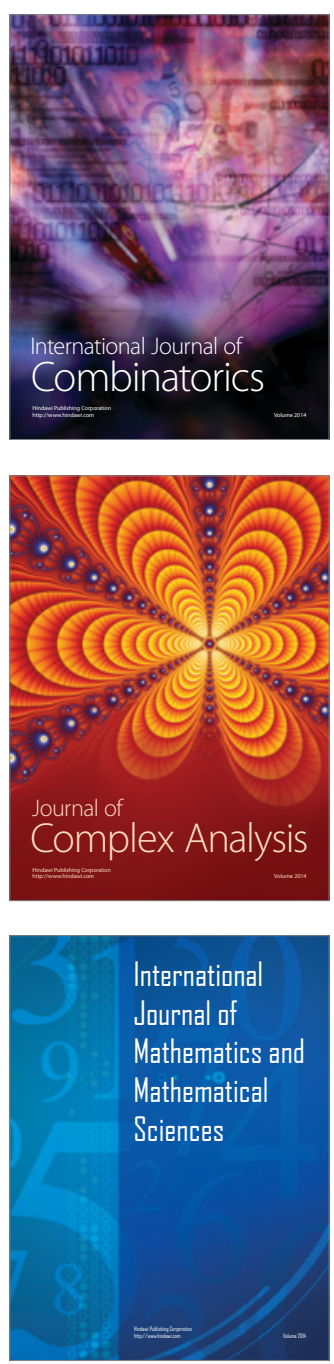
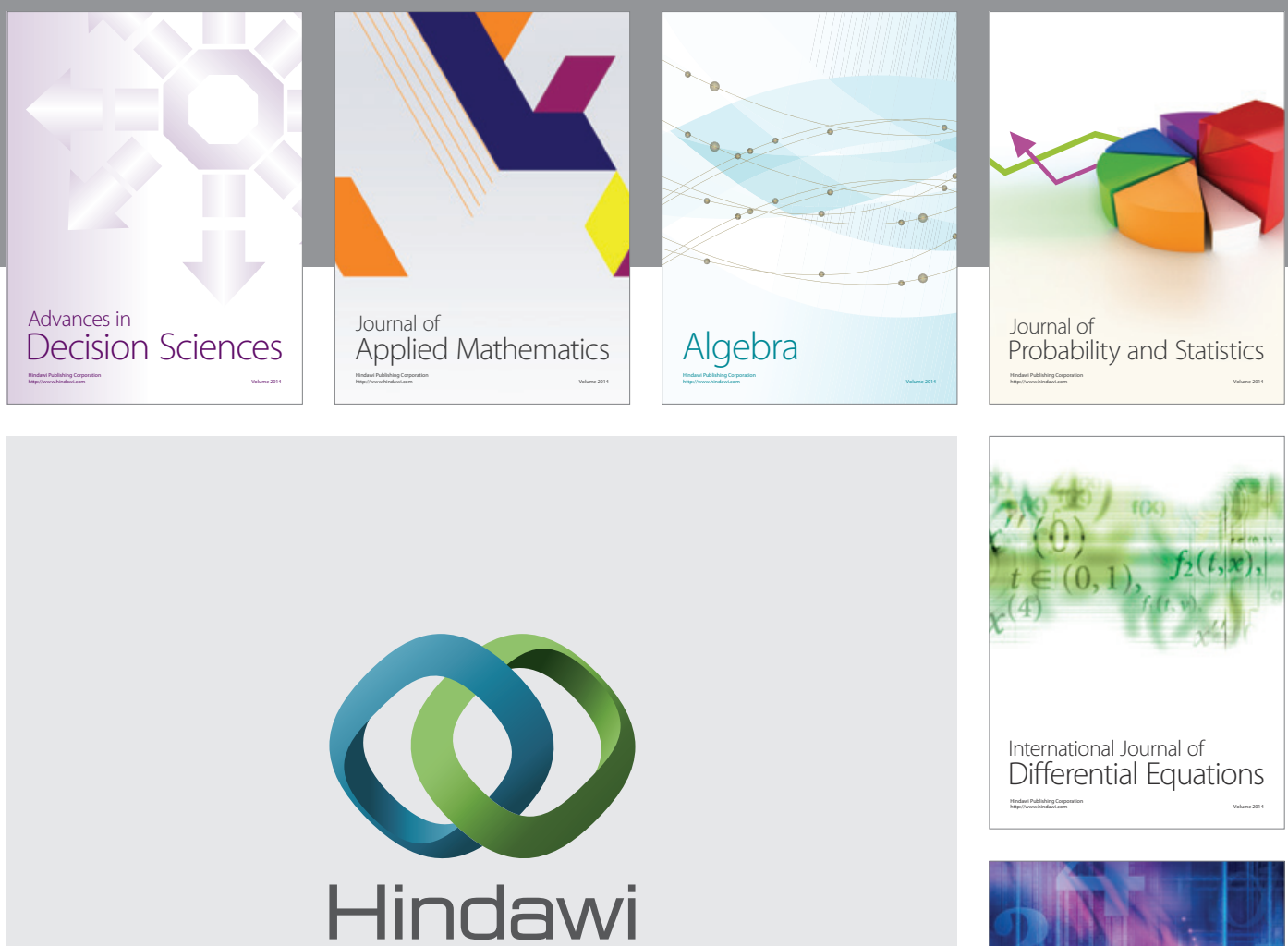

Submit your manuscripts at http://www.hindawi.com
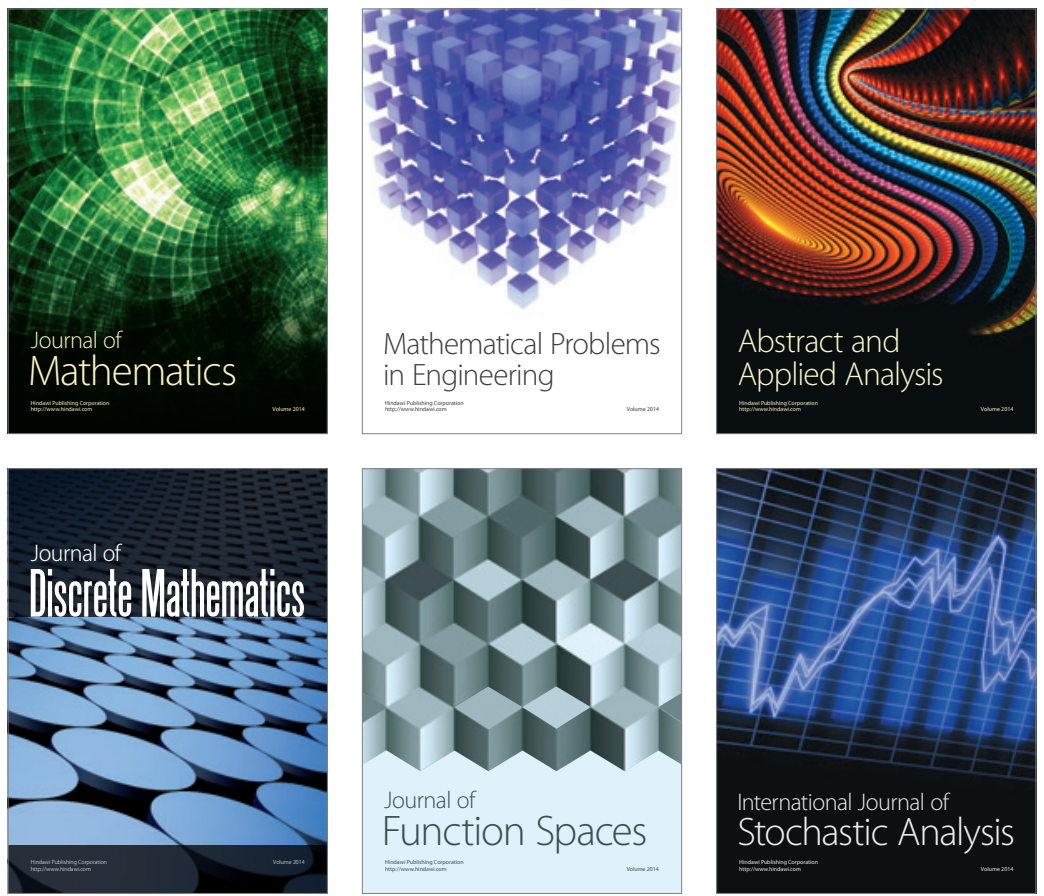

Journal of

Function Spaces

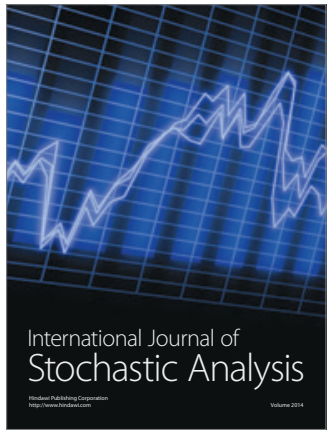

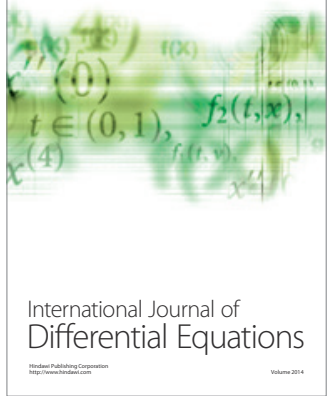
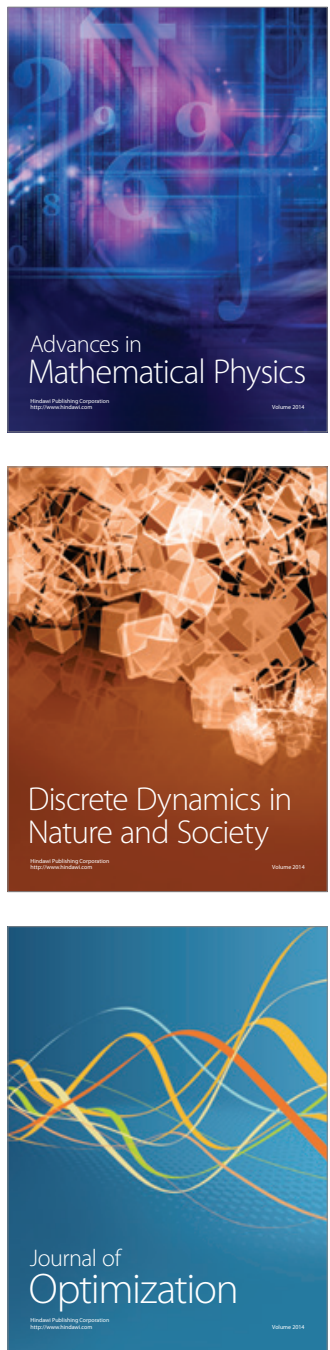